\title{
BMJ Open Protocol for a prospective observational study of cortical lower urinary tract control changes following intradetrusor injection of botulinum toxin-A in patients with multiple sclerosis
}

\author{
Rodolfo A Elizondo, ${ }^{1}$ Christof Karmonik, ${ }^{2}$ Timothy B Boone, ${ }^{3}$ Rose Khavari ${ }^{3}$
}

To cite: Elizondo RA, Karmonik C, Boone TB, et al. Protocol for a prospective observational study of cortical lower urinary tract control changes following intradetrusor injection of botulinum toxin-A in patients with multiple sclerosis. BMJ Open 2017;7:e013225. doi:10.1136/bmjopen-2016013225

- Prepublication history for this paper is available online. To view these files please visit the journal online (http://dx.doi.org/10.1136/ bmjopen-2016-013225)

Received 28 June 2016 Revised 29 December 2016 Accepted 4 January 2017

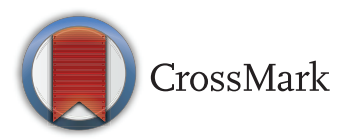

${ }^{1}$ Department of Pediatric Urology, Texas Children's Hospital, Houston, Texas, USA

${ }^{2}$ Department of MRI Core, Methodist Research Institute, Houston, Texas, USA

${ }^{3}$ Department of Urology,

Methodist Hospital, Houston, Texas, USA

Correspondence to

Dr Rose Khavari;

rkhavari@houstonmethodist. org

\section{ABSTRACT}

Introduction: Multiple sclerosis (MS) is a severe debilitating disease that affects patients' quality of life. Up to $90 \%$ of patients with MS will develop lower urinary tract dysfunction within the first 18 years of the disease. If oral pharmacotherapy with anticholinergics, behavioural modifications and pelvic floor physical therapy are unsuccessful, intradetrusor injection of botulinum toxin-A (OnaBotA; Botox Allergan, Dublin, Ireland) is a highly effective option for these patients. The local effects of OnaBotA are well understood, but not much is known of its afferent/sensory effects while treating the end organ. Our study will use functional MRI (fMRI) and task-related blood oxygen leveldependent signals to evaluate patients with MS and neurogenic detrusor overactivity (ND0) prior to, and after, intradetrusor injection of OnaBotA with simultaneous urodynamic evaluation. Urinary concentration of brain-derived neurotrophic factor and nerve growth factor will also be collected since it has been shown that patients with an overactive bladder have higher concentrations of these neuropeptides. Methods and analysis: Female patients with MS and lower urinary tract symptoms who previously have undergone urodynamic screening and are refractory to conservative and oral pharmacotherapy management for NDO and are interested in OnaBotA intradetrusor injection will be invited to participate in the study. An fMRI will be performed preintradetrusor injection and postintradetrusor injection of OnaBotA with simultaneous MRI compatible with material urodynamics. Images will be collected and analysed accordingly.

Ethics and dissemination: All of the patients are properly consented before enrolling in this study that has been previously approved by the Institutional Review Board. Results of neural connectivity activation will be presented at national and international meetings and published in scholarly journals.

\section{INTRODUCTION}

Multiple sclerosis (MS) is a chronic multifocal demyelinating disease that can affect any part of the central nervous system

\section{Strengths and limitations of this study}

- Identifying activation/deactivation of specific regions of the brain in patients with multiple sclerosis (MS) and neurogenic detrusor overactivity preintradetrusor injection and postintravesical injection of botulinum toxin-A (OnaBotA) using functional MRI during the entire micturition cycle: filling and voiding.

- Simultaneous correlation of the blood oxygen level-dependent signals with urodynamic tracings.

- Potentially identifying sensory effects of intradetrusor OnaBotA injection that could reveal a novel mechanism of action for OnaBotA used in the bladder.

- A specific neurogenic bladder patient population is being studied in this proposal and these findings cannot be generalised to all patients with a neurogenic bladder.

- Also, given that MS produces multifocal lesions in the brain, not all of the patients will have the same lesions in the brain or spinal cord and symptom severity will vary from patient to patient.

(CNS). Up to $90 \%$ of patients with MS will develop lower urinary tract (LUT) dysfunction within the first 18 years of the disease. ${ }^{12}$ Classification of voiding dysfunction is based on the degree of inability to store and/or empty urine. ${ }^{3}$ LUT symptoms (LUTS) experienced by patients with MS can range from urgency to urge urinary incontinence and/or hesitancy and incomplete bladder emptying. Owing to the diffuse, multifocal involvement of the CNS in patients with MS, symptom severity may vary from patient to patient. Approximately $70 \%$ of patients with MS report that their LUTS have a moderate-to-severe impact on their quality of life. ${ }^{4}$ Urgency, frequency and neurogenic 
detrusor overactivity (NDO) are the most common urological findings (34-99\%) during diagnostic evaluations of patients with MS. ${ }^{5}$ Even though anticholinergic or $\beta$ agonist drugs have limited effectiveness and adverse side effects, they are the first-line pharmacotherapy for patients with NDO if behavioural modifications and pelvic floor physical therapy are unsuccessful. ${ }^{6}$ Botulinum toxin-A (OnaBotA; Botox Allergan, Dublin, Ireland) intradetrusor injection is a highly effective treatment option for patients with NDO who are refractory to more conservative management. ${ }^{7-9}$

OnaBotA blocks the release of acetylcholine at the neuromuscular junction and leads to a temporary chemodenervation of the bladder (paralysis of the muscle). Motor effects of OnaBotA on the bladder have been extensively studied and widely reported in the literature, and the US Food and Drug Administration has approved OnaBotA for the treatment of detrusor overactivity in neurogenic and non-neurogenic patients. However, the sensory effects of OnaBotA injection correlating to CNS regional perception/localisation of urgency, frequency and urge incontinence in humans are not well known.

Only a few spinal cord injury animal models have suggested central inhibitory effects of OnaBotA. Animal studies have shown that the emergence of $\mathrm{C}$ fibres causes bladder overactivity with induction of non-voiding contractions in rats with spinal injury. Boone and colleagues have published their observations of the effect on central inhibition of intravesical instillation of OnaBotA in animals with spinal cord injury. They were able to show that OnaBotA-treated groups demonstrated a significant decrease in L6 (ie, 67\%, p<0.001) and S1 (ie, $47 \%, \mathrm{p}<0.01)$ c-fos expression $(43 \%)$ compared with controls receiving saline solution. This finding demonstrated that intravesical instillation of BXT-A significantly inhibits the response of bladder afferent activated lumbosacral neurons without significantly impairing efferent bladder function. ${ }^{10}$ In other animal models of bladder overactivity (ie, SCI and chronic bladder inflammation), intravesical instillation of OnaBotA has also been shown to selectively reduce the bladder's afferent response without significantly affecting efferent function (ie, bladder contractility). ${ }^{11}{ }^{12}$ Moreover, some studies support the idea of a potential role of sensory purinergic pathways in the development of bladder overactivity and suggest that OnaBotA can reduce purinergic transmission. ${ }^{13}{ }^{14}$ Whether or not these OnaBotA effects extend to the brain level remains to be elucidated.

Physiological control of urine storage and micturition relies on a complex network of neural circuits throughout the brain, spinal cord and urothelium. ${ }^{15}$ For a long time, animal models have been used to study LUT function, but with regard to lower urinary function, they differ in numerous ways (many of them social) from humans. Critical distinctions exist between voluntary voiding (humans) and involuntary leakage of urine (animal models) when all the social, emotional and mechanical criteria for voiding in humans are considered. This distinct intention to void or not to void highlights the need for human research in the area of CNS and LUTS instead of reliance mainly on animal data. The initial afferent stimulus comes from the sensation in the bladder as the desire to void. Afterwards, the forebrain determines a person's social circumstance and whether to proceed with the voiding stimulus. Once it is socially acceptable to void, centres in the brain and spinal cord coordinate to produce bladder contraction and urethral sphincter relaxation. ${ }^{15}{ }^{16}$ In order for the pontine micturition centre (PMC) to begin micturition, visceral sensations from passive filling in the bladder are transmitted to the periaqueductal grey (PAG) matter along with other structures in the brain such as the thalamus, insula and anterior cingulate gyrus. Additional neural structures associated with the voiding reflex are the motor prefrontal cortex, supplementary motor cortex and parahippocampus. ${ }^{17-19}$ All of these structures may be involved during PAG activation to transmit further input to the PMC to start micturition. ${ }^{20}{ }^{21}$ As noted earlier, patients with MS have multifocal lesion involvement of the CNS including the brain and spinal cord. The severity and presentation of the LUTS depends on the location of the lesions. ${ }^{2} 22$

Over the past decades, functional MRI (fMRI) has been used to study the activation of supraspinal LUT control centres in healthy participants during the storage and voiding phases. ${ }^{17-19} 2324$ Previous studies have shown that fMRI with simultaneous video urodynamic testing is feasible and can be used to evaluate or stratify treatment modalities for chronic pelvic pain or urge urinary incontinence. ${ }^{25}$ Furthermore, a German group investigated structural brain changes in women after they had undergone 12 weeks of treatment with physical therapy or biofeedback for stress urinary incontinence. $^{26}$ In this study, the investigators identified BOLD responses in primary motor and sensory cortical areas associated with pelvic floor muscles. In a study recently reported by Griffiths et $a l l^{20}$ the investigators identified two patterns of brain activation sensitive to bladder filling that could predict the response and nonresponse to pelvic floor physical therapy that targeted urinary urge incontinence. These data suggest that treatments targeting the end organ (bladder) can significantly affect CNS neuroplasticity. Given these facts, we are interested in evaluating the role of intradetrusor injection of OnaBotA afferent response in patients with MS and NDO.

Using fMRI, our group has already demonstrated the activation of brain networks consisting of interconnected regions of sensorimotor control (cerebellum, thalamus, caudate, lentiform nucleus, red nucleus, supplementary motor area, postcentral gyrus), executive function (left superior frontal gyrus) and emotion processing (anterior and posterior cingulate gyrus and insula), as well as deep brain structures (parahippocampal gyrus, percuneus, cuneus, occipital lobe and pons) during micturition in healthy women. ${ }^{24}$ We have also shown that 
patients with MS have distinct supraspinal control activation/deactivation patterns during the micturition cycle when compared with healthy controls. ${ }^{27}$ This can be the result of increased bladder afferent input to the brain. High-resolution neuroimaging techniques will help us understand how MS affects the bladder-brain controls over micturition. Our study will use fMRI and task-related blood oxygen level-dependent (BOLD) signals to evaluate patients with MS and NDO prior to, and after, intradetrusor injection of OnaBotA with simultaneous urodynamic evaluation. Urinary concentration of brain-derived neurotrophic factor (BDNF) and nerve growth factor (NGF) will also be collected since it has been shown that patients with an overactive bladder have higher concentrations of these neuropeptides. ${ }^{28}$ In the following, we will describe our previously established protocol, which we will use for evaluating brain activity with fMRI during the storage and voiding phases with a simultaneous urodynamic study (UDS). ${ }^{24}$

\section{Study aims}

1. To perform fMRI neuroimaging and data analysis in patients with MS and NDO during the act of filling and voiding, preintravesical injection and postintravesical injection of OnaBotA and to identify specific regions of the brain that are activated and/or deactivated in this group of patients.

2. To determine whether concurrent UDS data obtained with fMRI can be correlated to fMRI data in order to elucidate simultaneous bladder function measurements with BOLD signal measurements in this patient population.

3. To evaluate the role of urinary biomarkers associated with bladder overactivity (BDNF and NGF) in patients with MS and NDO, before and after intravesical injection of OnaBotA.

4. To determine whether the common validated urgency questionnaires correlate with fMRI findings and urinary biomarker concentration preinjection and postinjection of OnaBotA in patients with MS and NDO.

\section{METHODS AND ANALYSIS}

\section{Patient selection and recruitment}

We propose an observational prospective research study that has been approved by our Institutional Review Board prior to patient selection. Inclusion criteria consist of adult female patients with clinically stable MS for $\geq 3$ months before screening and an Expanded Disability Status Score (EDSS) $\leq 6.5$ with LUTS (urinary frequency or urgency) who previously have undergone a clinic urodynamic screening and are refractory to conservative and oral pharmacological management for NDO and are interested in OnaBotA intradetrusor injection, will be invited to participate in the study. Men will be excluded from the study to avoid confounding by prostate pathology (table 1). Other exclusion criteria
Table 1 Inclusion and exclusion criteria for participants

\begin{tabular}{|c|c|}
\hline Inclusion & Exclusion \\
\hline 18 years or older & Non-ambulatory \\
\hline Female & $\begin{array}{l}\text { Severe debilitating disease } \\
\text { (MS) }\end{array}$ \\
\hline $\begin{array}{l}\text { Patients with stable } \\
M S \geq 3 \text { months before } \\
\text { screening }\end{array}$ & Urinary tract infection \\
\hline $\begin{array}{l}\text { Neurogenic detrusor } \\
\text { overactivity }\end{array}$ & Positive pregnancy test \\
\hline $\begin{array}{l}\text { LUTS (urinary frequency, } \\
\text { urgency) }\end{array}$ & $\begin{array}{l}\text { Contraindication for MRI (ie, } \\
\text { pacemaker, metal implants) }\end{array}$ \\
\hline $\begin{array}{l}\text { Positive clinic UDS for } \\
\text { neurogenic detrusor } \\
\text { overactivity }\end{array}$ & Male \\
\hline $\begin{array}{l}\text { Refractory to conservative } \\
\text { and oral pharmacotherapy }\end{array}$ & $\begin{array}{l}\text { History of incontinence } \\
\text { surgery (ie, sling, MMK, } \\
\text { Burch) } \\
\text { History of LUT surgery (ie, } \\
\text { urethral dilation) } \\
\text { Other neurological diseases } \\
\text { (ie, spinal cord injury, } \\
\text { myelomeningocele, } \\
\text { Parkinson disease) }\end{array}$ \\
\hline
\end{tabular}

include severe debilitating disease, pregnancy or planning to become pregnant, nursing, MRI contraindications, history of stress urinary incontinence surgery (eg, sling, urethral suspension), history of augmentation cystoplasty, and presence of other neurological diseases. Patients with active UTI can be treated and subsequently screened to determine their participation in this study (table 1). A healthy female cohort described in a previous report will be used as controls. ${ }^{24}$

Patient recruitment is performed at the Houston Methodist Neurourology Clinic in Houston, Texas, USA by three full-time fellowship-trained urologist in the field of neurourology and pelvic reconstructive surgery who work closely with MS neurologist from all institutions in the Texas Medical Center. RK serves as the current clinical director of the Neurourology Clinic and her practice is focused on patients with neurogenic bladder and pelvic reconstruction surgery in men and women. Patients will be recruited in our clinic and assessed by RK. Recruitment will be initiated in March 2016 and will conclude when we have data from 30 patients.

\section{Evaluation}

Each patient will provide a detailed history and undergo a complete physical examination. Each will complete the following questionnaires and assessments: Expanded Disability Status Scale and Incontinence Quality of Life (I-QOL), Patient Perception of Bladder Control (PPBC), Bladder Control Scale (BLCS), Pelvic Organ Prolapse Distress Inventory 6 (POPDI-6), Demographic Form, 
and MRI Safety Screening Questionnaire. Postvoidal residual (PVR) volume will be measured and a urine sample will be obtained for urinalysis and neuropeptide evaluation. A baseline video UDS will be obtained within a year prior to the baseline neuroimaging scan. Consent and enrolment agreement will be signed during the first visit (see figure 1). Patients will be seen a total of four times: initial visit, fMRI control and OnaBotA injection, follow-up in the clinic at 2 and 6 weeks following OnaBotA treatment for symptom evaluation, urinalysis and PVR volume. All questionnaires will be completed after OnaBotA treatment as well as a simple patientreported perception of their treatment benefit using a four-point treatment-based scale. If there are clinical signs of increased PVR volume or urinary retention, clean intermittent catheterisations (CIC) will be initiated.

\section{Simultaneous urodynamic testing and fMRI}

Prior to entering the MRI room, all patients will answer the institutional standard clinical questionnaire for MRI safety and excluded from the study if contraindications to the MRI are found. A patient will be asked to wear a gown to avoid image artefacts through ferromagnetic objects in their clothing. Prior to the study, the patient will be asked to void, and PVR volume will be measured through catheterisation. Double lumen $7 \quad \mathrm{Fr}$ MRI-compatible catheters will be placed in the bladder and rectum. A Phillips Ingenia 3.0T full body MRI with a standard 12 channel head coil will be used. Instructions to communicate using right hand signals representing 'strong desire', 'voiding' and 'voiding completed' are given to the patient before imaging. To avoid auditory or other extensive brain stimulation not connected to the voiding process, simple signs will be shown to the patient when filling of the bladder is begun and when filling is stopped. Also, in order to keep our noise-to-signal ratio low, all stimulators including any extra visual stimuli and the urodynamic machine will be removed from the MRI room. Total scan times are limited to $45 \mathrm{~min}$. After acquisition of a high-resolution three-dimensional (3D) brain scan for anatomical reference (turbo fast field, echo, isotropic 3D resolution of
$0.7 \mathrm{~mm}$ ), echo-planar imaging (EPI; repetition time $3 \mathrm{~s}$, in-plane resolution $3.4 \mathrm{~mm}$, slice thickness $4 \mathrm{~mm}$ ) during two functional tasks are performed to record the BOLD signal. First, instruction signals from the patient's right hand (tasks) are performed without actual voiding and scanned separately to obtain a baseline for fMRI activation caused from signalling alone. Afterwards, urodynamic testing and the voiding task are simultaneously initiated. The bladder is filled at $50 \mathrm{~mL} / \mathrm{min}$ with room temperature, sterile, normal saline solution until the participant indicates a strong desire to void. Filling will be stopped and participants will be asked to hold the urine for $30 \mathrm{~s}$. Afterwards, patients will be allowed to void into absorbent pads on the scanner table. To create a blockdesign fMRI task, this sequence will be repeated up to four times. Final catheterisable PVR volume will be recorded. This procedure will be performed before treatment and 6-10 weeks after intradetrusor injection of OnaBotA (see figure 2).

\section{Intradetrusor OnaBotA injection}

During the second visit, the patient will have a cystoscopy with intradetrusor injection of OnaBotA performed by RK. One hundred (in spontaneous voiders) or 200 (CIC dependent patients) units of OnaBotA will be injected throughout the bladder, including the trigone. Patients will be asked to void to obtain uroflow, if they are spontaneous voiders, and postvoid residual volume will be measured.

\section{Data analysis and statistics}

Power analysis

Careful application of power calculation is done in the early phases of designing a study to prevent spending time and money on an experiment that is underpowered. This is especially important when more costly studies such as the current one are being designed. However, various and limited methods exist to calculate the fMRI power.

A study from 2002 presented an approach for group analysis in an fMRI model while accounting for intraparticipant and interparticipant variability. It concluded that $\sim 12$ participants were required to achieve $80 \%$
Figure 1 Schematic

representation of the first, second and third clinic visits where data will be collected. The fourth clinic visit at 6 weeks will not include an fMRI. fMRI, functional MRI; PVR, postvoidal residual.

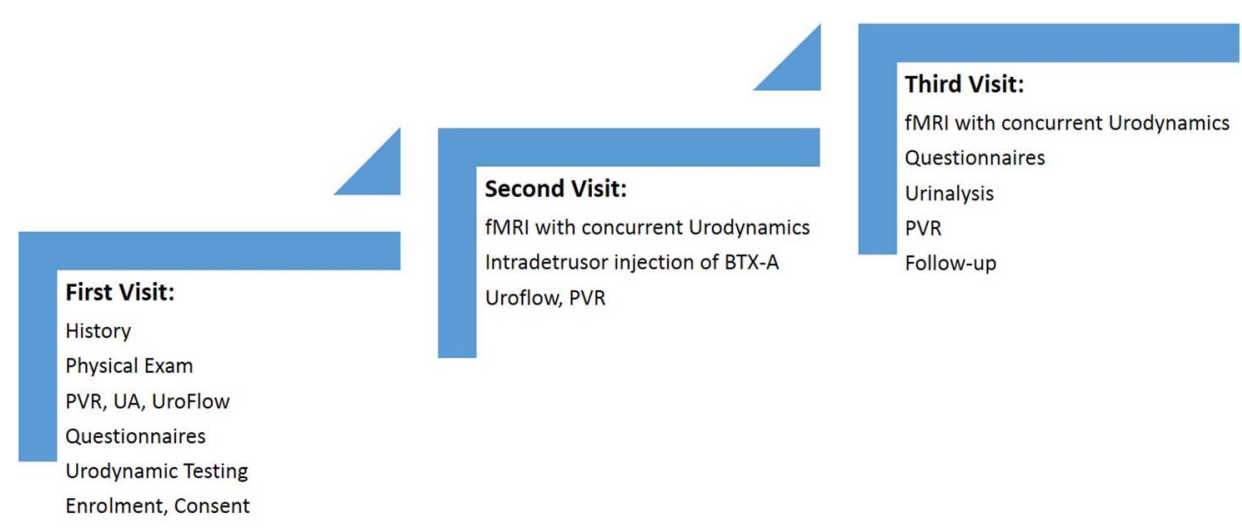


Figure 2 Flow chart of $\mathrm{FMRI}$ and a simultaneous urodynamic study. fMR, functional MRI; PVR, postvoidal residual.

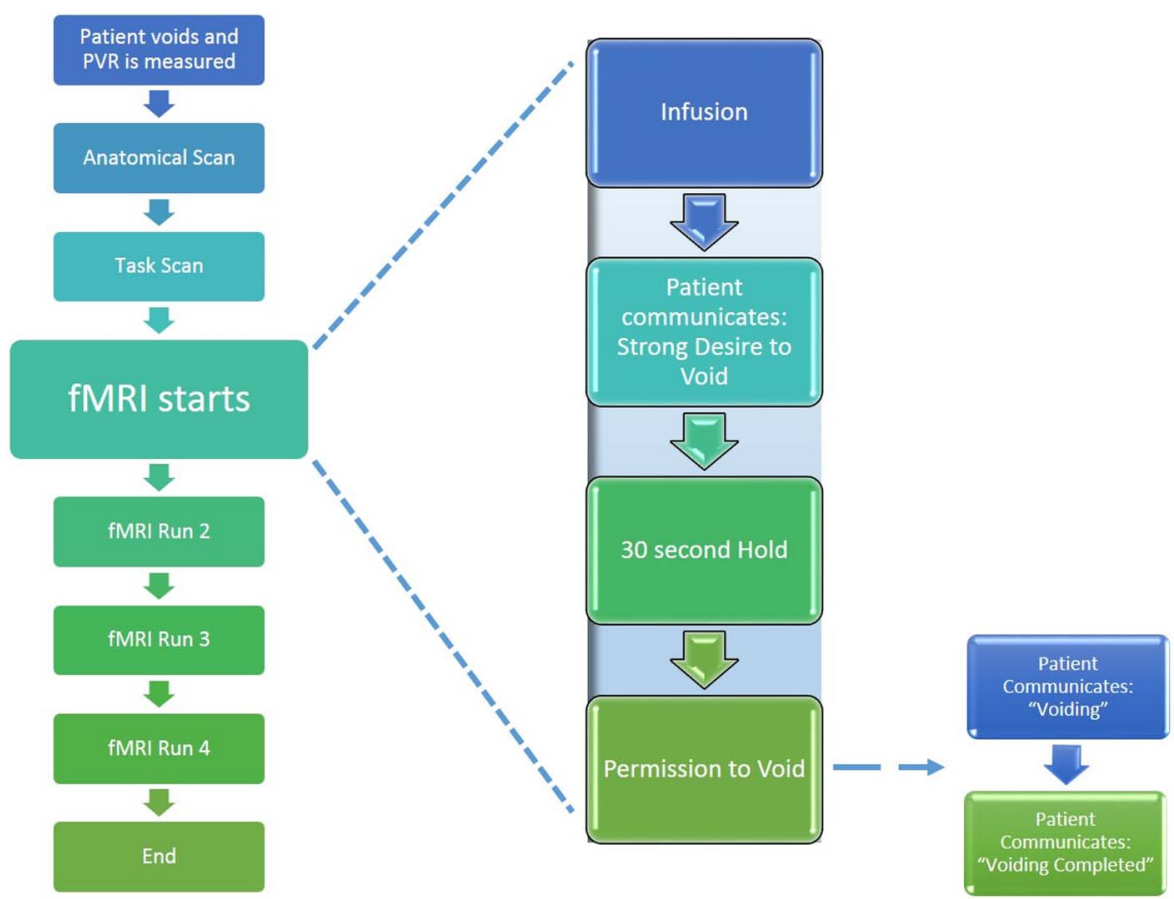

power at the single voxel level for typical activations. ${ }^{29}$ However, at more realistic thresholds after correcting for multiple comparisons, 24 participants should be used to maintain this level of power. A study by Murphy and colleagues performed an empirical investigation in data of 58 participants with go/no go studies. The correlation coefficients were calculated within the regions of interest (ROI) defined by the 58-participant map threshold at the strictest $p$ value of 0.000001 where there was a 0.8 correlation when 24 participants were included. ${ }^{30}$ To estimate the power of this study, we would take into account the $10 \%$ exclusions for movement artefacts and $20 \%$ dropout rates where patients may not return for post-OnaBotA scans and we concluded to study a total of 30 patients. The study will end when the 30th patient is scanned and evaluated after OnaBotA injection.

Three-dimensional structural images will be obtained from a T1-weighted sequence (sagittal direction, 0.7 in-plane resolution). Functional images will be collected afterwards by means of simultaneous urodynamic analysis (axial EPI, TR=3000 ms, $4.0 \mathrm{~mm}$ slice thickness, $3.38 \mathrm{~mm}$ in-plane resolution). AFNI software (NIMH, https://afni.nimh.nih.gov/afni/) will be used for the fMRI BOLD analysis. Structural and functional images will be registered to each other. The EPI fMRIs will be motion-corrected. Translational and rotational motion extent will be quantified in this step. Patients with large motion $(<4.5 \mathrm{~mm})$ will be excluded from analysis. Voxel activation will be identified at the time of 'strong desire to void' and 'the initiation voiding'; and BOLD fMRI activation maps will be calculated for both conditions using standard analysis with the generalised linear model. Group analysis will be performed by transforming data into the Talairach space, and significantly activated voxels will be identified using Student's t-test. Comparisons will be drawn between participants with MS comparing baseline scans to the post-OnaBotA treatment. Subgroup analysis will be performed comparing participants with and without detrusor sphincter dyssynergia. We will use SPSS (V.19.0) to perform statistical analysis of clinical data (ie, demographic, UDS parameters).

Functional connectivity analysis will be performed using CONN (NITRC https://www.nitrc.org/projects/ conn), a functional connectivity (FC) toolbox for MATLAB57, V.15a, which uses the statistical parametric mapping MATLAB toolbox. fMRIs acquired during the voiding task, together with their corresponding anatomical data sets, will be processed using the default-mni preprocessing option to enable group analysis in the Montreal Neurological Institute (MNI) space. A regionbased connectivity analysis will be performed for each patient group preinjection and postinjection of OnaBotA based on ROI $(n=4)$ defined in a brain atlas available from the toolbox. FC is the connectivity between brain regions that share functional properties. It can be defined as the temporal correlation between spatially remote neurophysiological events assessed by their respective BOLD signal time courses. FC will be quantified by $\mathrm{T}$ values for a $\mathrm{p}$ value $<0.05$ (two-sided, FDR-corrected).

Diffusion tensor imaging (DTI) will be acquired (32 directions, 1 B0 image) using the standard MRI pulse sequence available on the Philips 3.0 T Ingenia scanner. The original DTI, as well as the fractional anisotropy (FA) and mean diffusivity (MD) calculated on the scanner, will be transferred to an offline workstation for further processing. The software packages TackVis 
(V.0.6.0.1) and the Diffusion Toolkit (V.0.6.3, trackvis. org) will be used to calculate and extract selected white matter tracts of interest. This software also enables one to calculate FA and MD values for the segmented white matter tracts.

\section{Safety}

Every staff member involved in the collection of data and handling of the patient will be trained and evaluated regarding MRI safety before being allowed to enter the scanner room. Pregnancy tests and urinalysis will exclude patients who are pregnant or have a urinary tract infection. For the latter, after successful treatment of urinary tract infection, patients will be rescheduled.

\section{Limitations}

Since this study protocol focuses on a specific neurogenic bladder patient population, these findings cannot be generalised to all patients with a neurogenic bladder. Also, given that MS produces multifocal lesions in the brain, not all of the patients will have the same lesions in the brain or spinal cord and symptom severity will vary from patient to patient. This limiting factor will be thoroughly described when we present our results in a future manuscript submission.

\section{ETHICS AND DISSEMINATION}

Informed consent will be obtained before enrolling a patient in the study. Our informed consent states that participation is completely voluntary and patients can withdraw at any point and this will not affect their relationship with the physician and their treatment course. All staff members involved in the collection of data and handling of patients will have proper privileges and training by our research institute and MRI core. Before proceeding with fMRI testing, patients will be asked to remove all clothing and items containing metal. All materials used during the fMRI/UDS are MRI compatible. Participants will be provided with gowns during the imaging study. No minor or vulnerable individuals will be recruited for the study. If an adverse effect occurs, the principal investigator and appropriate authorities will be informed and proper actions will be taken based on good clinical practice. Outcomes of this research will be presented at national and international meetings and published in scholarly journals.

Contributors RAE and CK contributed to the writing and revision of the manuscript. TBB and RK contributed to the study design and manuscript revision.

Funding RK is a scholar supported in part by the National Institutes of Health (NIH) grant K12 DK0083014, the Multidisciplinary K12 Urologic Research (KURe) Career Development Program to Dolores J Lamb (DJL) from the National Institute of Diabetes and Digestive and Kidney Diseases (NIDDK), NIH. In addition, Society of Urodynamics, Female Pelvic Medicine, and Urological Reconstruction Research Foundation Award (Chemodenervation award) contributes to part of this project.

Competing interests None declared.

Ethics approval Approval of the Institutional Review Board (Houston Methodist Research Institute) will be obtained for all study procedures.
Provenance and peer review Not commissioned; externally peer reviewed.

Open Access This is an Open Access article distributed in accordance with the Creative Commons Attribution Non Commercial (CC BY-NC 4.0) license, which permits others to distribute, remix, adapt, build upon this work noncommercially, and license their derivative works on different terms, provided the original work is properly cited and the use is non-commercial. See: http:// creativecommons.org/licenses/by-nc/4.0/

\section{REFERENCES}

1. de Sèze $M$, Ruffion $A$, Denys $P$, et al. The neurogenic bladder in multiple sclerosis: review of the literature and proposal of management guidelines. Mult Scler Houndmills Basingstoke Engl 2007; 13:915-28.

2. Phé V, Chartier-Kastler E, Panicker JN. Management of neurogenic bladder in patients with multiple sclerosis. Nat Rev Urol 2016;13:275-88.

3. Wein AJ. Pathophysiology and classification of lower urinary tract dysfunction: overview. In: Campbel-Welsh: urology. US: Elsevier Health Science, 2016.

4. Fowler CJ, Panicker JN, Drake M, et al. A UK consensus on the management of the bladder in multiple sclerosis. J Neurol Neurosurg Psychiatr 2009;80:470-7.

5. Wein AJ, Dmochowski RR. Neuromuscular dysfunction of the lower urinary tract. In: Campbel-Welsh: urology. US: Elsevier Health Science, 2016.

6. Buser N, Ivic S, Kessler TM, et al. Efficacy and adverse events of antimuscarinics for treating overactive bladder: network meta-analyses. Eur Urol 2012;62:1040-60.

7. Leone Roberti Maggiore U, Salvatore S, Alessandri F, et al. Pharmacokinetics and toxicity of antimuscarinic drugs for overactive bladder treatment in females. Expert Opin Drug Metab Toxicol 2012;8:1387-408

8. Gormley EA, Lightner DJ, Faraday M, et al. Diagnosis and treatment of overactive bladder (non-neurogenic) in adults: AUA/SUFU guideline amendment. J Urol 2015;193:1572-80.

9. Yamaguchi O, Nishizawa O, Takeda M, et al. Clinical guidelines for overactive bladder. Int J Urol 2009;16:126-42.

10. Munoz A, Somogyi GT, Boone TB, et al. Central inhibitory effect of intravesically applied botulinum toxin $A$ in chronic spinal cord injury. Neurourol Urodyn 2011;30:1376-81.

11. Khera M, Somogyi GT, Salas NA, et al. In vivo effects of botulinum toxin A on visceral sensory function in chronic spinal cord-injured rats. Urology 2005;66:208-12.

12. Smith CP, Vemulakonda VM, Kiss S, et al. Enhanced ATP release from rat bladder urothelium during chronic bladder inflammation: effect of botulinum toxin A. Neurochem Int 2005;47:291-7.

13. Apostolidis A, Jacques TS, Freeman A, et al. Histological changes in the urothelium and suburothelium of human overactive bladder following intradetrusor injections of botulinum neurotoxin type A for the treatment of neurogenic or idiopathic detrusor overactivity. Eur Urol 2008;53:1245-53.

14. Cheng CL, Liu JC, Chang SY, et al. Effect of capsaicin on the micturition reflex in normal and chronic spinal cord-injured cats. Am J Physiol 1999;277:R786-94.

15. Chai TC, Birder LA. Physiology and pharmacology of the bladder and the urethra. US: Elsevier Health Science, 2016.

16. Griffiths DJ, Fowler CJ. The micturition switch and its forebrain influences. Acta Physiol Oxf Engl 2013;207:93-109.

17. Kuhtz-Buschbeck JP, Gilster R, van der Horst C, et al. Control of bladder sensations: an fMRI study of brain activity and effective connectivity. Neuroimage 2009;47:18-27.

18. Kuhtz-Buschbeck JP, van der Horst $C$, Wolff $S$, et al. Activation of the supplementary motor area (SMA) during voluntary pelvic floor muscle contractions-an fMRI study. Neuroimage 2007;35:449-57.

19. Kuhtz-Buschbeck JP, van der Horst C, Pott C, et al. Cortical representation of the urge to void: a functional magnetic resonance imaging study. J Urol 2005;174:1477-81.

20. Griffiths D, Clarkson B, Tadic SD, et al. Brain mechanisms underlying urge incontinence and its response to pelvic floor muscle training. J Urol 2015;194:708-15.

21. Panicker JN, Fowler CJ, Kessler TM. Lower urinary tract dysfunction in the neurological patient: clinical assessment and management. Lancet Neurol 2015;14:720-32.

22. Wintner A, Kim MM, Bechis SK, et al. Voiding dysfunction in multiple sclerosis. Semin Neurol 2016;36:219-20.

23. Krhut J, Tintera J, Holý $\mathrm{P}$, et al. A preliminary report on the use of functional magnetic resonance imaging with simultaneous urodynamics to record brain activity during micturition. J Urol 2012;188:474-9. 
24. Shy M, Fung S, Boone TB, et al. Functional magnetic resonance imaging during urodynamic testing identifies brain structures initiating micturition. J Urol 2014;192:1149-54.

25. Borghesi G, Simonetti R, Goldman SM, et al. Magnetic resonance imaging urodynamics. Technique development and preliminary results. Int Braz J Urol 2006;32:336-41; discussion 341.

26. Di Gangi Herms AM, Veit R, Reisenauer C, et al. Functional imaging of stress urinary incontinence. Neuroimage 2006;29:267-75.

27. Khavari R, Karmonik C, Shy M, et al. Functional magnetic resonance imaging with concurrent urodynamic testing identifies brain structures involved in micturition cycle in patients with multiple sclerosis. J Urol 2017;197:438-44.

28. Antunes-Lopes T, Pinto R, Barros SC, et al. Urinary neurotrophic factors in healthy individuals and patients with overactive bladder. $J$ Urol 2013:189:359-65.

29. Desmond JE, Glover GH. Estimating sample size in functional MRI (fMRI) neuroimaging studies: statistical power analyses. J Neurosci Methods 2002;118:115-28.

30. Murphy K, Garavan H. An empirical investigation into the number of subjects required for an event-related fMRI study. Neuroimage 2004;22:879-85. 